\title{
Research on the Horizontal Displacement Coefficient of Soil Surrounding Pile in Layered Foundations by Considering the Soil Mass's Longitudinal Continuity
}

\author{
Yao Wen-Juan ${ }^{1}$ and Zhou Hua-Cong ${ }^{1,2}$ \\ ${ }^{1}$ Department of Civil Engineering, Shanghai University, No. 149 Yanchang Road, Shanghai 200072, China \\ ${ }^{2}$ College of Architecture and Engineering, Anhui Polytechnic University, Wuhu 241000, China \\ Correspondence should be addressed to Yao Wen-Juan; wenjuan@mail.shu.edu.cn
}

Received 22 April 2013; Revised 21 June 2013; Accepted 24 June 2013

Academic Editor: Zhiqiang Hu

Copyright (C) 2013 Y. Wen-Juan and Z. Hua-Cong. This is an open access article distributed under the Creative Commons Attribution License, which permits unrestricted use, distribution, and reproduction in any medium, provided the original work is properly cited.

\begin{abstract}
When utilizing the $p-y$ curve to simulate the nonlinear characteristics of soil surrounding pile in layered foundations, due to having not taken into account the soil mass's longitudinal continuity, the calculation deviation of horizontal displacement increases with the growth of a load. This paper adopted the layered elasticity system theory to consider the soil mass's longitudinal continuity, as well as utilizing the research method for layered isotropic bodies, assuming that the horizontal resistance is evenly distributed around the perimeter of the pile's cross-section. Then an appropriate transfer matrix method of horizontal displacement coefficient for the soil surrounding pile in layered foundations was established. According to the calculation principle of finite element equivalent load, the horizontal displacement coefficient matrix was deduced as well as providing a corrected formula for the horizontal displacement of soil surrounding pile through the $p-y$ curve method when the external load was increased. Following the established model, a program was created which was used for calculating and analyzing the horizontal displacement coefficient matrix of three-layered soil in order to verify this method's validity and rationale. Where there is a relatively large discrepancy in the soil layers' properties, this paper's method is able to reflect the influence on the layered soil's actual distributional difference as well as the nearby soil layers' interaction.
\end{abstract}

\section{Introduction}

For combining the $p-y$ curve method with the finite element method, it can be applicable to layered foundation systems and able to take the soil's nonlinear characteristics into consideration. Several scholars [1-11] have carried out the simulation of single pile's load-bearing characteristics and improved the $p-y$ curves method of piles under lateral loads or lateral and axial loads. Whereas with the $p-y$ curve method, when hypothesizing that the $p-y$ curves of different depths are unrelated to each other, this actually results in the soil's longitudinal continuity being overlooked. When the horizontal load is gradually increased merely by using the $p-y$ curve combined with the finite element method and other numerical methods to calculate the displacement of soil surrounding pile, the deviation between the calculated results and the measured engineering value, or the simulated results, will gradually enlarge. Therefore, the calculations of the $p-y$ curve method need to be amended when the horizontal load is large to make up for the insufficiencies in the soil mass's longitudinal continuity.

The method which takes into consideration the soil mass's longitudinal continuity is proposed in the analysis theory of elasticity. It calculates the displacement of soil surrounding pile at the node by the Mindlin solution as the relevant horizontal displacement coefficient when the soil is undergoing unit load; then the corresponding matrix of the horizontal displacement coefficient can be obtained and be used for the next step of calculations or amendments. However, the elasticity theory's solution for horizontal 
displacement coefficient is based on the Mindlin solution for homogeneous soil, as well as only being suitable for the situation of the concentrated load acting upon the half space and can only be applied to solve horizontal displacement coefficients in homogenous soil or equivalent. Most research [12-18] has developed on the foundation of axisymmetrical space, whereas related research regarding the problem of nonaxisymmetric space has been less, especially on the effect caused by the horizontal resistance of the soil surrounding pile towards the layered elastic foundation.

This paper takes each layer of soil mass as simply being an isotropic elastomer, regards layer foundations as isotropic elastomers, and assumes that the horizontal resistance is evenly distributed around the perimeter of the pile's crosssection. This paper takes the transfer matrix method as its basis [19] in line with elastic foundation theory and layered elasticity systems theory and presents a solution applicable for the finite element method's horizontal displacement coefficient matrix for soil surrounding pile as well as providing revisions to the matrix expression of horizontal displacement of soil surrounding pile. At the same time, in order to avoid the exponential overflow problem of the transfer matrix method's numerical calculations, this paper introduces the precise integration method (PIM) [20] to calculate the layered elastic foundation's dynamic stiffness matrix.

Speaking of layered soil and regarding the traditional $p-y$ curve method for horizontal load-bearing piles, the improved method is the general Mindlin solution which only considers the axisymmetric problem, and the solution in the elasticity theory for horizontal displacement coefficients is based on the Mindlin solution for homogenous soils. The new thinking in this paper is to consider not only the nonaxisymmetric problem but also the layered character and nonlinearity of the soil.

\section{Transfer Matrix Expression for Homogenous Infinite Half Space}

The displacement functions of non-axisymmetric space are $\varphi=\varphi(r, \theta, z)$ and $\psi=\psi(r, \theta, z)$. The relationship between displacements $u, v$, and $w$ and the displacement functions $\varphi$ and $\psi$ is as follows [21]:

$$
\begin{gathered}
u=-\frac{1+\mu}{E}\left(\frac{\partial^{2} \varphi}{\partial r \partial z}-\frac{2}{r} \frac{\partial \psi}{\partial \theta}\right), \\
v=-\frac{1+\mu}{E}\left(\frac{1}{r} \frac{\partial^{2} \varphi}{\partial \theta \partial z}-2 \frac{\partial \psi}{\partial r}\right), \\
w=\frac{1+\mu}{E}\left[2(1-\mu) \nabla^{2} \varphi-\frac{\partial^{2} \varphi}{\partial z^{2}}\right] .
\end{gathered}
$$

In (1), $u, v$, and $w$ are the displacements, $\varphi$ and $\psi$ is the displacement function, $E$ is the Young's modulus, $\mu$ is the Poisson's ratio, and $\nabla^{2}=\partial^{2} / \partial x^{2}+\partial^{2} / \partial y^{2}+\partial^{2} / \partial z^{2}$.
According to the generalized Hooke law and the displacement function (1), the series expansion for displacements $u, v$, and $w$ can also be expressed as

$$
\begin{aligned}
& u=\frac{1+\mu}{E} \sum_{k=0}^{\infty} u_{k}(r, z) \cdot \cos k \theta, \\
& v=\frac{1+\mu}{E} \sum_{k=0}^{\infty} v_{k}(r, z) \cdot \sin k \theta, \\
& w=\frac{1+\mu}{E} \sum_{k=0}^{\infty} w_{k}(r, z) \cdot \cos k \theta .
\end{aligned}
$$

In (2), $r, \theta$, and $z$ are the cylindrical coordinates.

Substituting it into the elasticity equilibrium equation, we get

$$
\begin{gathered}
\sigma_{r}=\sum_{k=0}^{\infty} \sigma_{r k} \cos k \theta, \\
\sigma_{\theta}=\sum_{k=0}^{\infty} \sigma_{\theta k} \cos k \theta, \\
\sigma_{z}=\sum_{k=0}^{\infty} \sigma_{z k} \cos k \theta, \\
\tau_{r \theta}=\sum_{k=0}^{\infty} \tau_{r \theta k} \sin k \theta, \\
\tau_{\theta z}=\sum_{k=0}^{\infty} \tau_{\theta z k} \sin k \theta, \\
\tau_{z r k}=\sum_{k=0}^{\infty} \tau_{z r k} \cos k \theta .
\end{gathered}
$$

Equations (2), (3), and (4) are the expressions of the transfer matrix method's displacement and stress progression. Substituting the previous three equations into equilibrium and geometric equations, we get

$$
\begin{gathered}
\frac{\partial \sigma_{r}}{\partial r}+\frac{k}{r} \tau_{r \theta k}+\frac{\partial \tau_{z r k}}{\partial z}+\frac{\sigma_{r k}-\sigma_{\theta k}}{r}=0, \\
\frac{\partial \tau_{r \theta k}}{\partial r}+\frac{k}{r} \sigma_{\theta k}+\frac{\partial \tau_{\theta z k}}{\partial z}+\frac{2}{r} \tau_{r \theta k}=0, \\
\frac{\partial \tau_{z r k}}{\partial r}+\frac{k}{r} \tau_{\theta z k}+\frac{\partial \sigma_{z k}}{\partial z}+\frac{1}{r} \tau_{z r k}=0, \\
\sigma_{r k}=\frac{1}{1-2 \mu}\left\{\left[(1-\mu) \frac{\partial}{\partial r}+\frac{\mu}{r}\right] u_{k}+\frac{\mu k}{r} v_{k}+\mu \frac{\partial \omega_{k}}{\partial z}\right\}, \\
\sigma_{\theta k}=\frac{1}{1-2 \mu}\left[\left(\mu \frac{\partial}{\partial r}+\frac{1-\mu}{r}\right) u_{k}+\frac{(1-\mu) k}{r} v_{k}+\mu \frac{\partial \omega_{k}}{\partial z}\right], \\
\sigma_{r k}=\frac{1}{1-2 \mu}\left[\mu\left(\frac{\partial}{\partial r}+\frac{1}{r}\right) u_{k}+\frac{\mu k}{r} v_{k}+(1-\mu) \frac{\partial \omega_{k}}{\partial z}\right],
\end{gathered}
$$




$$
\begin{gathered}
\tau_{r \theta k}=\frac{1}{2}\left[-\frac{\mu}{r} u_{k}+\left(\frac{\partial}{\partial r}-\frac{1}{r}\right) v_{k}\right], \\
\tau_{\theta z k}=\frac{1}{2}\left(\frac{\partial v_{k}}{\partial z}-\frac{k}{r} \omega_{k}\right), \\
\tau_{z r k}=\frac{1}{2}\left(\frac{\partial u_{k}}{\partial z}+\frac{\partial \omega_{k}}{\partial r}\right) .
\end{gathered}
$$

In (6), $u_{k}$ and $v_{k}$ are the layer displacements, in which

$$
\begin{gathered}
\tau_{1}=\tau_{\theta z k}+\tau_{z r k}, \\
\tau_{2}=\tau_{\theta z k}-\tau_{z r k}, \\
U_{k}=u_{k}+v_{k}, \\
V_{k}=u_{k}-v_{k} .
\end{gathered}
$$

Furthermore,

$$
\begin{aligned}
& X(\xi, z)=\left[\sigma_{z k(\xi, z)} ; \tau_{1(\xi, z)} ; \tau_{2(\xi, z)} ; U_{k(\xi, z)} ; V_{k(\xi, z)} ; \omega_{k(\xi, z)}\right], \\
& X(\xi, 0)=\left[\sigma_{z k(\xi, 0)} ; \tau_{1(\xi, 0)} ; \tau_{2(\xi, 0)} ; U_{k(\xi, 0)} ; V_{k(\xi, 0)} ; \omega_{k(\xi, 0)}\right], \\
& \bar{X}(\xi, z)=\left[\bar{\sigma}_{z k(\xi, z)} ; \bar{\tau}_{1(\xi, z)} ; \bar{\tau}_{2(\xi, z)} ; \bar{U}_{k(\xi, z)} ; \bar{V}_{k(\xi, z)} ; \bar{\omega}_{k(\xi, z)}\right], \\
& \bar{X}(\xi, 0)=\left[\bar{\sigma}_{z k(\xi, 0)} ; \bar{\tau}_{1(\xi, 0)} ; \bar{\tau}_{2(\xi, 0)} ; \bar{U}_{k(\xi, 0)} ; \bar{V}_{k(\xi, 0)} ; \bar{\omega}_{k(\xi, 0)}\right] .
\end{aligned}
$$

In $(8), \bar{X}(\xi, z)$ is the stress and displacement component in random layer and $\bar{X}(\xi, 0)$ is the stress and displacement component in the surface layer.

In addition, the fundamental relation between the Hankel transform and the inverse transform is

$$
\begin{aligned}
& \bar{X}(\xi, z)=\int_{0}^{\infty} r X(\xi, z) J_{n}(\xi r) d r ; \\
& X(\xi, z)=\int_{0}^{\infty} \xi \bar{X}(\xi, z) J_{n}(\xi r) d \xi .
\end{aligned}
$$

In (9), $J_{n}(\xi r)$ is the Bessel function and $\xi$ is the scaling factor of the Bessel functions.

Undergoing basic transform and the Hankel integral transform, (5) and (6) can be stated as

$$
\begin{gathered}
\frac{d \bar{\sigma}_{z k}}{d z}=-\frac{\xi}{2}\left(\bar{\tau}_{1}+\bar{\tau}_{2}\right) \\
\frac{d \bar{\tau}_{1}}{d z}=-\frac{\xi}{2(1-\mu)}\left\{2 \mu \cdot \bar{\sigma}_{z k}+\frac{\xi}{2}\left[(3-\mu) \bar{U}_{k}-(1+\mu) \bar{V}_{k}\right]\right\} \\
\frac{d \bar{\tau}_{2}}{d z}=-\frac{\xi}{2(1-\mu)}\left\{2 \mu \cdot \bar{\sigma}_{z k}+\frac{\xi}{2}\left[(1+\mu) \bar{U}_{k}-(3-\mu) \bar{V}_{k}\right]\right\} \\
\frac{d \bar{U}_{k}}{d z}=2 \bar{\tau}_{1}+\xi \bar{\omega}_{k} \\
\frac{d \bar{V}_{k}}{d z}=-\left(2 \bar{\tau}_{2}+\xi \bar{\omega}_{k}\right) \\
\frac{d \bar{\omega}_{k}}{d z}=-\frac{1}{1-\mu}\left[(1-2 \mu) \cdot \bar{\sigma}_{z k}-\frac{\mu \xi}{2}\left(\bar{U}_{k}-\bar{V}_{k}\right)\right] .
\end{gathered}
$$

In (10), $\tau_{1}$ and $\tau_{2}$ are the shear stresses. Equation (10) can be phrased as the matrix differential equation

$$
\frac{d[\overline{\mathbf{X}}]}{d z}=[\mathbf{A}][\overline{\mathbf{X}}] .
$$

Solving the matrix differential equation (11), the transfer matrix expression for within homogenous infinite half space can be obtained when the soil thickness or load transfer distance is $z_{i}$ :

$$
\bar{X}(\xi, z)=\left[\mathbf{G}\left(\xi, z_{i}\right)\right] \bar{X}(\xi, 0) .
$$

In (12), $\left[\mathbf{G}\left(\xi, z_{i}\right)\right]$ is the transfer matrix [15] for surfaceto-depth $z_{i}$ of a single-layer semi-infinite system, expressed as follows:

$$
\begin{aligned}
\left\{\begin{array}{c}
\bar{\sigma}_{z k}(\xi, z) \\
\bar{\tau}_{1}(\xi, z) \\
\bar{\tau}_{2}(\xi, z) \\
\bar{U}_{k}(\xi, z) \\
\bar{V}_{k}(\xi, z) \\
\bar{\omega}_{k}(\xi, z)
\end{array}\right\}= & {\left[\begin{array}{llllll}
G_{11} & G_{12} & G_{13} & G_{14} & G_{15} & G_{16} \\
G_{21} & G_{22} & G_{23} & G_{24} & G_{25} & G_{26} \\
G_{31} & G_{32} & G_{33} & G_{34} & G_{35} & G_{36} \\
G_{41} & G_{42} & G_{43} & G_{44} & G_{45} & G_{46} \\
G_{51} & G_{52} & G_{53} & G_{54} & G_{55} & G_{56} \\
G_{61} & G_{62} & G_{63} & G_{64} & G_{65} & G_{66}
\end{array}\right] } \\
& \times\left\{\begin{array}{c}
\bar{\sigma}_{z k}(\xi, 0) \\
\bar{\tau}_{1}(\xi, 0) \\
\bar{\tau}_{2}(\xi, 0) \\
\bar{U}_{k}(\xi, 0) \\
\bar{V}_{k}(\xi, 0) \\
\bar{\omega}_{k}(\xi, 0)
\end{array}\right\} .
\end{aligned}
$$

\section{Expression and Transformation for Horizontal Load on a Pile Shaft}

The form for distribution of horizontal resistance of the soil surrounding pile and the concentrated force form as adopted in the Mindlin solution, or the form for circular distribution and hyperbolic distribution as adopted in pavement engineering, have large differences in their distribution patterns. To simplify the pile-soil model and make it easier to elicit the analytical formula, this paper assumed that the horizontal resistance of the pile shaft is evenly distributed around the perimeter of the pile's cross-section and selected the passive soil pressure in the compression side of the pile-soil interface as the horizontal resistance of soil surrounding pile, as shown in Figure 1.

The expression for horizontal loads is as follows:

$$
p(r)=\left(\frac{P}{2 \pi r_{0}}\right) \cdot \delta\left(r-r_{0}\right) .
$$

In (14), $r_{0}$ is the pile's diameter, $P$ is the total load on the perimeter of the pile's cross-section, and $P$ is a constant. $\delta(r-$ $\left.r_{0}\right)$ is the Dirac function. Regarding this equation's 0-order Hankel transform and combining with the Dirac function's characteristics, it becomes

$$
\begin{aligned}
\bar{p}(r) & =\int_{0}^{\infty} r \cdot\left(\frac{P}{2 \pi r_{0}}\right) \cdot \delta\left(r-r_{0}\right) \cdot J_{0}(\xi r) d r \\
& =r_{0} \cdot \frac{P}{2 \pi r_{0}} \cdot J_{0}\left(\xi r_{0}\right)=\frac{P}{2 \pi} \cdot J_{0}\left(\xi r_{0}\right) .
\end{aligned}
$$




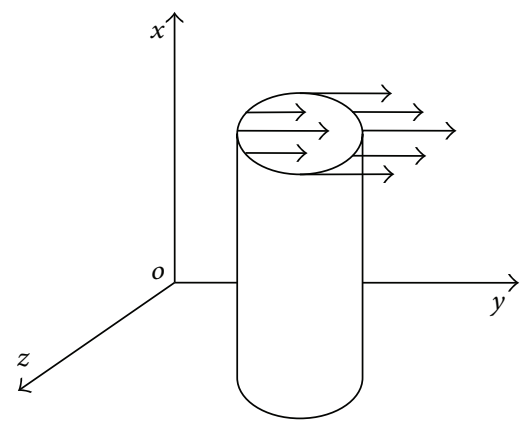

Figure 1: Assumption of distribution of horizontal resistance.

During analysis of a single pile, the direction of $p(r)$ is $\theta=0^{\circ}$ when setting the particular coordinate axis. Then, the variations of horizontal load application point are

$$
\begin{gathered}
\left.\Delta \tau_{\theta z}\right|_{z=z_{p}}=p(r) \sin \theta, \\
\left.\Delta \tau_{z r}\right|_{z=z_{p}}=-p(r) \cos \theta .
\end{gathered}
$$

Amongst which, $z_{p}$ is the horizontal loading depth. According to the solution characteristics of soil resistance, during the process of solving the horizontal displacement coefficient, we assume that there is no pile in the soil mass. According to (4)'s trigonometric series transform, (16) is translated into the unit force acting on the pile as the internal force of the layered system. Then, using the Hankel transform, we get

$$
\Delta \bar{X}\left(\xi, z_{p}\right)=\Delta \overline{\mathbf{P}}\left(\xi, z_{p}\right)=\left[\begin{array}{llllll}
0 & 0 & -2 \bar{p}(r) & 0 & 0 & 0
\end{array}\right]^{T} .
$$

\section{Transfer Matrix Solution for Horizontal Load Acting on the Layered Foundation}

4.1. Interlayer Contact Conditions. The layered foundation's characteristics are shown in Figure 2, where $E_{s n}$ and $\mu_{s n}$ are the soil's elasticity modulus and Poisson's ratio in corresponding layer, and $h_{s n}$ is the corresponding soil thickness. The horizontal load acts on the interior of the layered soil mass.

Assuming that each layer's complete continuity, that is, the layer displacement and stress distribution continuity, then the $s n$ layer and $s n+1$ layer's contact conditions are

$$
\begin{gathered}
\left.\sigma_{z(s n)}\right|_{z=H(s n)}=\left.\sigma_{z(s n+1)}\right|_{z=H(s n)}, \\
\left.\tau_{\theta z(s n)}\right|_{z=H(s n)}=\left.\tau_{\theta z(s n+1)}\right|_{z=H(s n)}, \\
\left.\tau_{z r(s n)}\right|_{z=H(s n)}=\left.\tau_{z r(s n+1)}\right|_{z=H(s n)}, \\
\left.u_{(s n)}\right|_{z=H(s n)}=\left.u_{(s n+1)}\right|_{z=H(s n)}, \\
\left.v_{(s n)}\right|_{z=H(s n)}=\left.v_{(s n+1)}\right|_{z=H(s n)}, \\
\left.w_{(s n)}\right|_{z=H(s n)}=\left.w_{(s n+1)}\right|_{z=H(s n)} .
\end{gathered}
$$

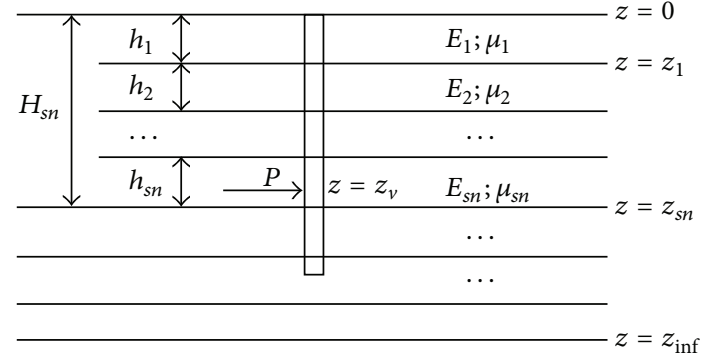

FIGURE 2: Horizontal load acting internally on the layered soil mass.

In (18), $H_{s n}$ is the layer's depth of contact point and $H(s n)=\sum_{i=1}^{s n} h_{s n}$. According to (2), (3), and (4), we use the Hankel transform for layer's contact conditions in (18) and get

$$
\begin{aligned}
\bar{\sigma}_{z k}\left(\xi, z_{s n-}\right) & =\bar{\sigma}_{z k}\left(\xi, z_{s n+}\right), \\
\bar{\tau}_{1}\left(\xi, z_{s n-}\right) & =\bar{\tau}_{1}\left(\xi, z_{s n+}\right), \\
\bar{\tau}_{2}\left(\xi, z_{s n-}\right) & =\bar{\tau}_{2}\left(\xi, z_{s n+}\right), \\
\frac{E_{s n}}{1+\mu_{s n}} \bar{U}_{k}\left(\xi, z_{s n-}\right) & =\frac{E_{s n+1}}{1+\mu_{s n+1}} \bar{U}_{k}\left(\xi, z_{s n+}\right), \\
\frac{E_{s n}}{1+\mu_{s n}} \bar{V}_{k}\left(\xi, z_{s n-}\right) & =\frac{E_{s n+1}}{1+\mu_{s n+1}} \bar{V}_{k}\left(\xi, z_{s n+}\right), \\
\frac{E_{s n}}{1+\mu_{s n}} \bar{\omega}_{k}\left(\xi, z_{s n-}\right) & =\frac{E_{s n+1}}{1+\mu_{s n+1}} \bar{\omega}_{k}\left(\xi, z_{s n+}\right) .
\end{aligned}
$$

In (19), the top and bottom surfaces are separately expressed by $z_{s n+1}$ and $z_{s n-1}$ for the corresponding soil depth $z_{s n}$. The remaining subscript patterns are similar.

4.2. Deducing the Transfer Formula of Transfer Matrix Method. When the horizontal load application point is $z=$ $z_{v}$, as on the foundation of (12), then, according to (19) for the layers' displacement stress continuity condition, the whole layered foundation system can be considered after being split into two parts. Amongst this, the first part is from $z_{\text {inf }}$ to $z_{v+}$, and the second part is from $z_{v-}$ to $z=0 . z_{v+}$ expresses the initial point for downwards calculation of the depth $z_{v}$ and $z_{v-}$ expresses the initial point for upwards calculation of the depth $z_{v}$. The matrix transfer formula from $z=0$ to the bottom is

$$
\begin{gathered}
\bar{X}\left(\xi, z_{v-}\right)=[G]_{z v-} \cdots\left\{\left[\mathrm{MC}_{0}\right][G]_{0}\right\} \bar{X}(\xi, 0) \\
\bar{X}\left(\xi, z_{v+}\right)=[G]_{z v-} \cdots\left\{\left[\mathrm{MC}_{0}\right][G]_{0}\right\} \bar{X}(\xi, 0)+\bar{P}_{z v} \\
\bar{X}\left(\xi, z_{v+}\right)=[G]_{i+1} \cdots\left\{\left[\mathrm{MC}_{0}\right][G]_{0}\right\} \bar{X}(\xi, 0) \\
+[G]_{i+1}\left\{\left[\mathrm{MC}_{i}\right][G]_{i}\right\} \\
\cdots\left\{\left[\mathrm{MC}_{z v+1}\right][G]_{z v+1}\right\} \bar{P}_{z v} .
\end{gathered}
$$


In the previous equations, $\bar{P}_{z v}$ is the stress and displacement array increment of the horizontal load application point, and

$$
[\mathrm{MC}]_{s n}=\left[\begin{array}{cccccc}
1 & 0 & 0 & 0 & 0 & 0 \\
0 & 1 & 0 & 0 & 0 & 0 \\
0 & 0 & 1 & 0 & 0 & 0 \\
0 & 0 & 0 & m_{s n} & 0 & 0 \\
0 & 0 & 0 & 0 & m_{s n} & 0 \\
0 & 0 & 0 & 0 & 0 & m_{s n}
\end{array}\right]
$$

Amongst which, $m_{s n}=E_{s n+1}\left(1+\mu_{s n}\right) / E_{s n}\left(1+\mu_{s n+1}\right)$, and $m_{0}=1$.

4.3. Definite Conditions. In practical engineering, the sufficiently far stress, deformation, and displacement can be defined as 0 . For superlong piles, due the pile top to the piletip distance being rather far, the effect of the horizontal load is extremely small. Therefore, the horizontal displacement of the pile tip can be defined as 0 . Also for the superlong piles, principally for friction piles or frictional end-bearing piles, the settlement value of the pile tip can be defined as 0 or a fixed constant. To simplify the solution, the definite condition of the pile tip can be defined as

$$
U_{k}\left(\xi, z_{l}\right)=V_{k}\left(\xi, z_{l}\right)=\omega_{k}\left(\xi, z_{l}\right)=0 .
$$

That is,

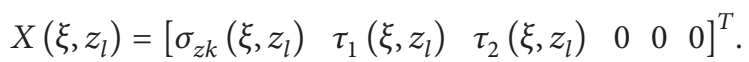

Amongst which, $z_{l}$ is the depth of the buried pile.

Due to there being no load on the soil surface when $z=0$, the boundary condition can be defined as

$$
\bar{X}(\xi, 0)=\left[\begin{array}{llllll}
0 & 0 & 0 & \bar{U}_{k}(\xi, 0) & \bar{V}_{k}(\xi, 0) & \bar{\omega}_{k}(\xi, 0)
\end{array}\right]^{T} .
$$

According to the two boundary conditions of (23) and (24), the actual force conditions of the layered foundation system cannot be fully reflected. According to (17), where $z=z_{v}, \bar{P}_{z v}$ can be represented as

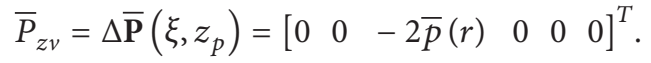

\section{Deducing the Horizontal Displacement Coefficient Matrix}

Supposing that the foundation is a layered isotropic elastomer, the displacement of soil surrounding pile can then be obtained by the generalized Mindlin solution under horizontal load. During the calculation process in the finite element method, the pile shaft can be divided into a number of units.

In Figure 3(a), the pile shaft's node numbering is $1 \sim n$. Due to the interaction between the pile and the soil, the soil's horizontal displacement $U_{i}$ for node $i$ can be represented as

$$
U_{i}=u_{1}+u_{2} \text {. }
$$

Amongst which, $u_{1}$ is the horizontal displacement produced by the equivalent horizontal resistance at node $i$, and $u_{2}$ is the horizontal displacement produced by the equivalent horizontal resistance at other nodes. This equation can also be represented as

$$
U_{i}=P_{i} u_{i, i}^{e}+\left(\sum_{j=0}^{i-1} P_{j} u_{i, j}^{e}+\sum_{j=i+1}^{n} P_{j} u_{i, j}^{e}\right) .
$$

In (27), $\{\mathbf{P}\}$ is the horizontal soil resistance array, and $u_{i, j}^{e}$ represents the horizontal displacement at node $i$ produced by the equivalent horizontal resistance acting at node $j$, which is the element in line $i$ and column $j$ in the horizontal displacement coefficient matrix. Consider

$$
\left[\overline{\mathbf{u}}^{e}\right]=\left[\begin{array}{ccccc}
u_{1,1}^{e} & u_{2,1}^{e} & \cdots & u_{(n-1), 1}^{e} & u_{n, 1}^{e} \\
u_{1,2}^{e} & u_{2,2}^{e} & \cdots & u_{(n-1), 2}^{e} & u_{n, 2}^{e} \\
\vdots & \vdots & \ddots & \vdots & \vdots \\
u_{1,(n-1)}^{e} & u_{2,(n-1)}^{e} & \cdots & u_{(n-1),(n-1)}^{e} & u_{n,(n-1)}^{e} \\
u_{1, n}^{e} & u_{2, n}^{e} & \cdots & u_{(n-1), n}^{e} & u_{n, n}^{e}
\end{array}\right]
$$

The horizontal displacement array $\{\mathbf{U}\}$ of soil surrounding pile is

$$
\{\mathbf{U}\}=\left[\overline{\mathbf{u}}^{e}\right]_{n \times n}\{\mathbf{P}\} .
$$

When the pile shaft is undergoing a horizontal load, the horizontal resistance around the pile will actually have continuous distribution. Every two continuous elements undergoing the horizontal resistance are shown in Figure 3(b). Regarding the distribution load, the finite element method often adopts the following equation to calculate the node's equivalent load:

$$
\mathbf{P}^{(e)}=\int_{0}^{L} \mathbf{N}^{T} q(x) l d \xi .
$$

In (30), $\mathbf{P}^{(e)}$ is the horizontal equivalent load, $\mathbf{N}$ is the displacement shape function, $q(x)$ is the horizontal distributed load, and $l$ is the pile length.

During the process of calculating the horizontal resistance of node $i$, the continuous distribution of soil resistance within the scope of two adjacent nodes $i-1$ and $i+1$ has been considered. Therefore, (27) can be rewritten as

$$
U_{i}=u_{1}^{\prime}+\sum_{j=0}^{i-2} P_{j} u_{e j i}+\sum_{j=i+2}^{n} P_{j} u_{e j i}
$$

In (31), $u_{1}^{\prime}$ is the horizontal displacement produced by the equivalent horizontal resistance of node $i$ and adjacent nodes $i-1$ and $i+1$, and it can be calculated by the $p-y$ curve method. $\left[\mathbf{u}^{e}\right]$ is the horizontal displacement coefficient matrix of the rewritten form. Consider

$$
\left[\mathbf{u}^{e}\right]=\left[\overline{\mathbf{u}}^{e}\right]-\left[\begin{array}{ccccc}
u_{1,1}^{e} & u_{2,1}^{e} & & & 0 \\
u_{1,2}^{e} & u_{2,2}^{e} & \ddots & & \\
& u_{2,3}^{e} & \ddots & u_{(n-1),(n-2)}^{e} & \\
& & \ddots & u_{(n-1),(n-1)}^{e} & u_{n,(n-1)}^{e} \\
0 & & & u_{(n-1), n}^{e} & u_{n, n}^{e}
\end{array}\right]
$$




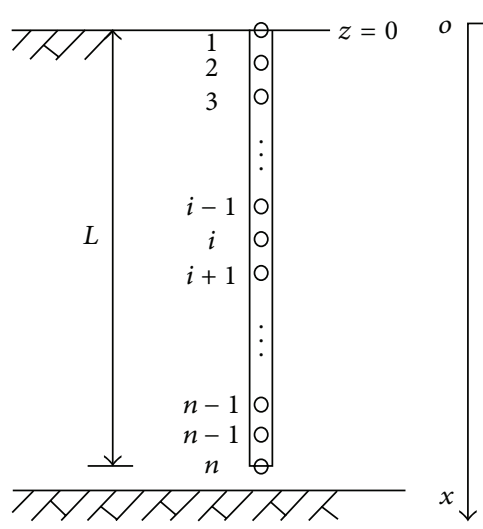

(a)

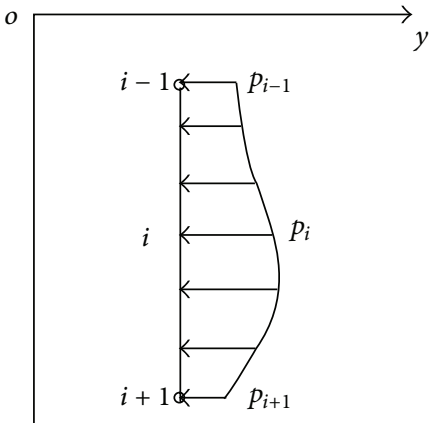

(b)

FIgURE 3: Pile foundation model.

According to the $p$ - $y$ curve method or other methods, the calculation of horizontal displacement for soil mass is

$$
\left[\mathbf{u}_{i}\right]=\left[\begin{array}{lllll}
u_{1,1}^{e} & u_{2,2}^{e} & \cdots & u_{(n-1),(n-1)}^{e} & u_{n, n}^{e}
\end{array}\right]^{T}
$$

Under a larger load, the horizontal displacement array $\{\mathbf{U}\}$ of soil surrounding pile can be expressed as

$$
\{\mathbf{U}\}=\left[\mathbf{u}_{i}\right]+\left[\mathbf{u}^{e}\right]\{\mathbf{P}\} .
$$

\section{The Solving Process of Horizontal Displacement Coefficient Matrix}

Based on the layered elasticity systems theory, the arithmetic solution of the horizontal displacement coefficient matrix $\left[u^{e}\right]_{n \times n}$ can be obtained by the transfer matrix method. The solving process is shown in Figure 4.

\section{Selecting the $p-y$ Curve}

The $p$ - $y$ curve of Hohai University that we selected in this paper reflects the actual situation of engineering and tallies well with the experimental results [22]. The coefficient is also relatively easy to determine. The $p-y$ curve is as follows:

$$
\begin{gathered}
p=\frac{y / y_{50}}{a+b y / y_{50}} p_{u} \frac{y}{y_{50}} \leq \beta, \\
p=p_{u} \quad \frac{y}{y_{50}}>\beta .
\end{gathered}
$$

Amongst which, $a=\beta /(\beta-1), b=(\beta-2) /(\beta-1), y_{50}=$ $4.5 \varepsilon_{50} D^{0.75}, \beta=\varepsilon_{100} / \varepsilon_{50}, \varepsilon_{50}$ is the strain value that is equal to a half of the difference stress in the triaxial undrained compressing test.

The ultimate soil resistance $p_{u}$ is as follows:

$$
\begin{gathered}
p_{u}=\left(3+\frac{\gamma}{C_{u}} z+\frac{J}{D} z\right) C_{u} D, \\
p_{u}=9 C_{u} D .
\end{gathered}
$$

Amongst which, $C_{u}$ is the triaxial undrained shear strength, $\gamma$ is the average severe, $z$ is the depth, $D$ is the pile diameter, and $J$ is the experience coefficient, usually $J=0.5$.

\section{Confirmation and Analysis of the Example}

A certain single pile at a dock is buried to soil depth of $36 \mathrm{~m}$ with a diameter $D=1 \mathrm{~m}$. The soil surrounding pile is divided into three layers. The surface layer thickness is $2 \mathrm{~m}$, the second layer thickness is $4 \mathrm{~m}$, and the third layer extends infinitely. The three layers are identical with Poisson's ratios of 0.3 . In order to more clearly perceive the results, the modulus is taken as a dimensionless number. Two cases are discussed as follows:

(1) $E_{1}=1$. The modulus ratios are $E_{1}: E_{2}: E_{3}=1: 1: 1$, $E_{1}: E_{2}: E_{3}=1: 2: 3, E_{1}: E_{2}: E_{3}=1: 3: 2$ and $E_{1}: E_{2}: E_{3}=1: 3: 5$.

(2) $E_{3}=1$. The modulus ratios are $E_{1}: E_{2}: E_{3}=1: 1: 1$, $E_{1}: E_{2}: E_{3}=3: 2: 1, E_{1}: E_{2}: E_{3}=2: 3: 1$ and $E_{1}: E_{2}: E_{3}=5: 3: 1$.

Using the paper's method and the classic Mindlin formula, respectively, the horizontal displacement coefficient for soil surrounding pile within the depth range of $0-36 \mathrm{~m}$ can be calculated and comparisons made. In order to increase comparability, as well as to avoid the horizontal displacement coefficient from trending towards an infinitely large situation, (28) is adopted for the transfer matrix method's results. The horizontal displacement coefficient's average value of the points on the pile's perimeter cross-section is taken as the arithmetic solution of the Mindlin formula.

According to (34), the horizontal displacement coefficient of this example can be directly used to amend the horizontal displacement of soil surrounding pile.

When the soil's modulus is $E_{1}: E_{2}: E_{3}=1: 1: 1$, the results of the horizontal displacement coefficient for unit load acting at the depth of $5 \mathrm{~m}$ are shown in Figure 5, and the comparison between the transfer matrix method's result and the finite element method's result is shown in Figure 6. 


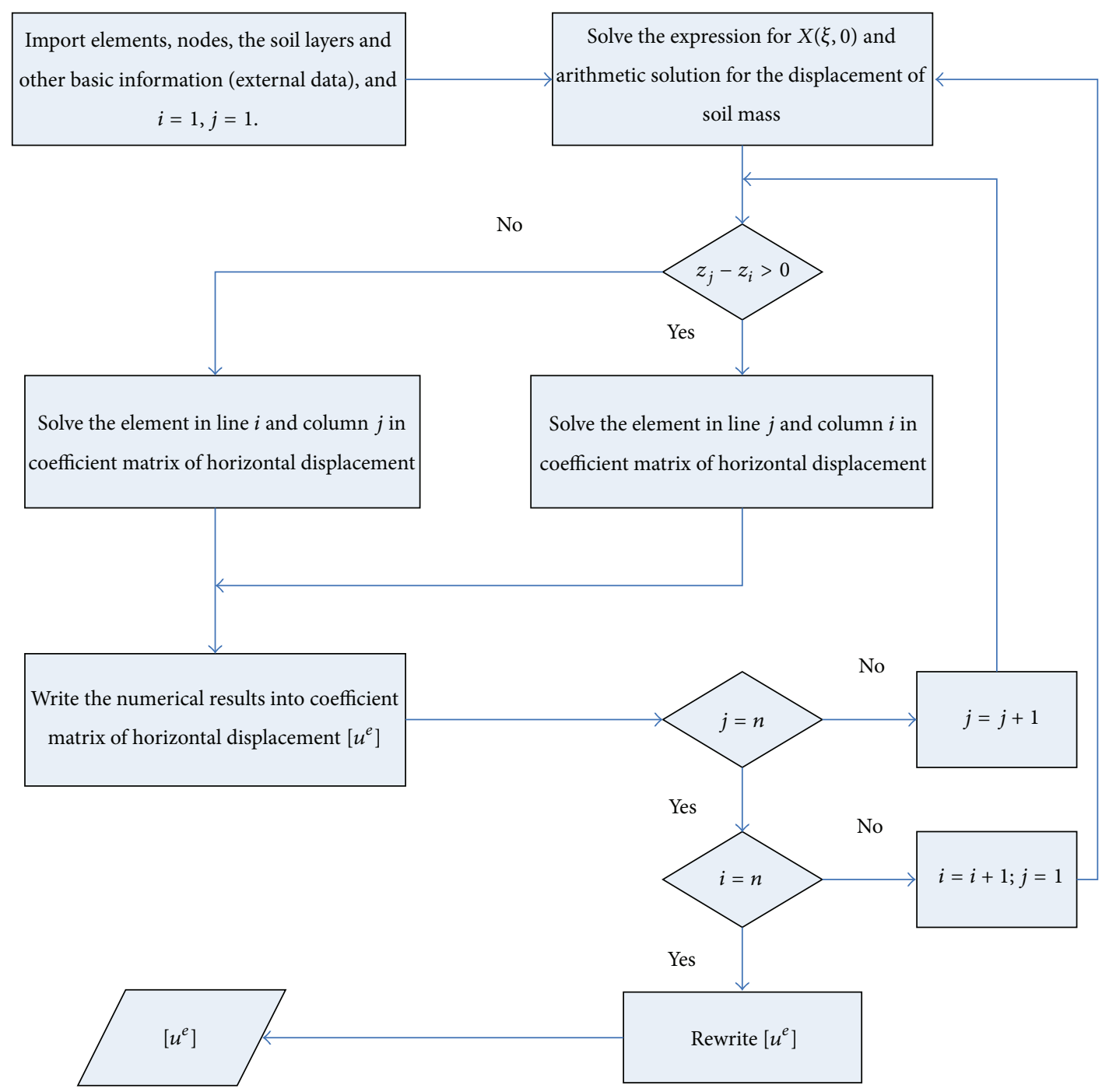

Figure 4: Calculation flowchart.

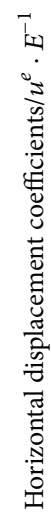
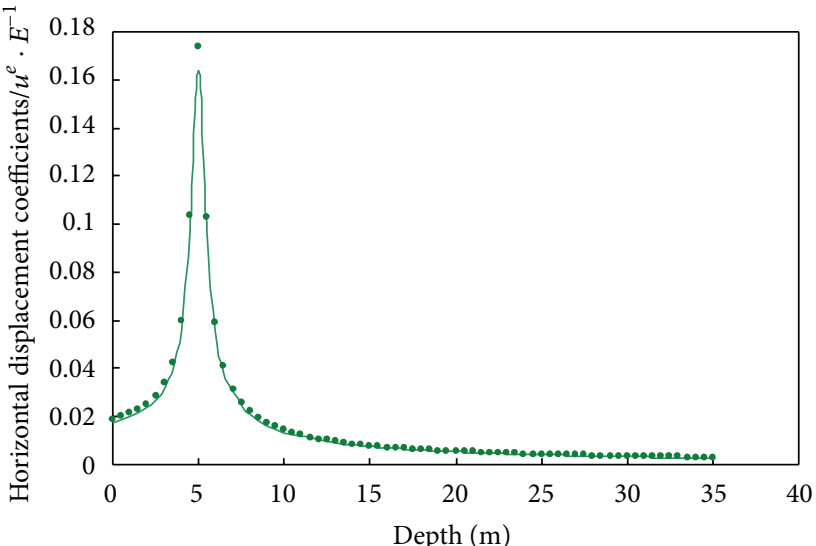

$E_{1}: E_{2}: E_{3}=1: 1: 1$

- Mindlin

Figure 5: Comparison between the transfer matrix method and the Mindlin solution (unit load acting at $5 \mathrm{~m}$ ).

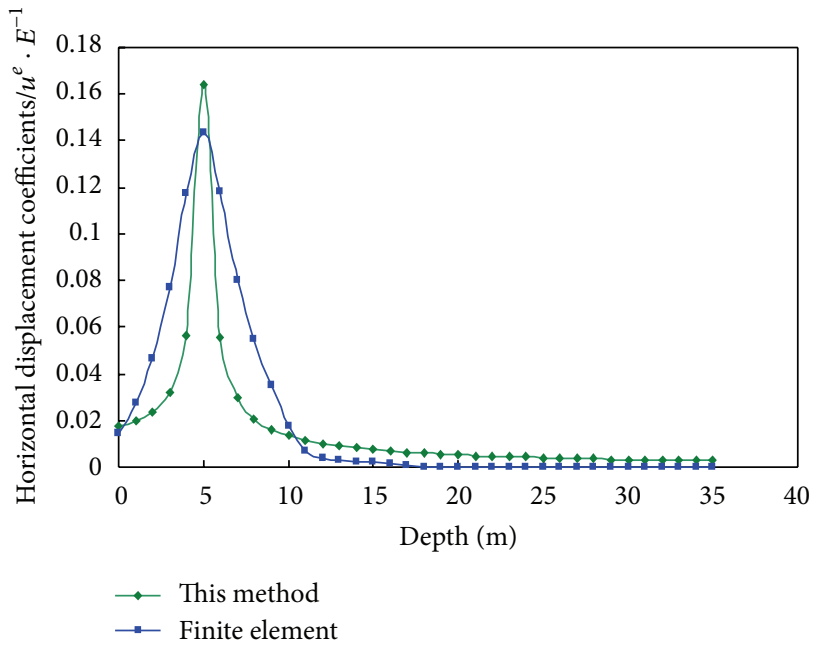

FIGURE 6: Comparison between the transfer matrix method and the finite element method (unit load acting at $5 \mathrm{~m}$ ). 


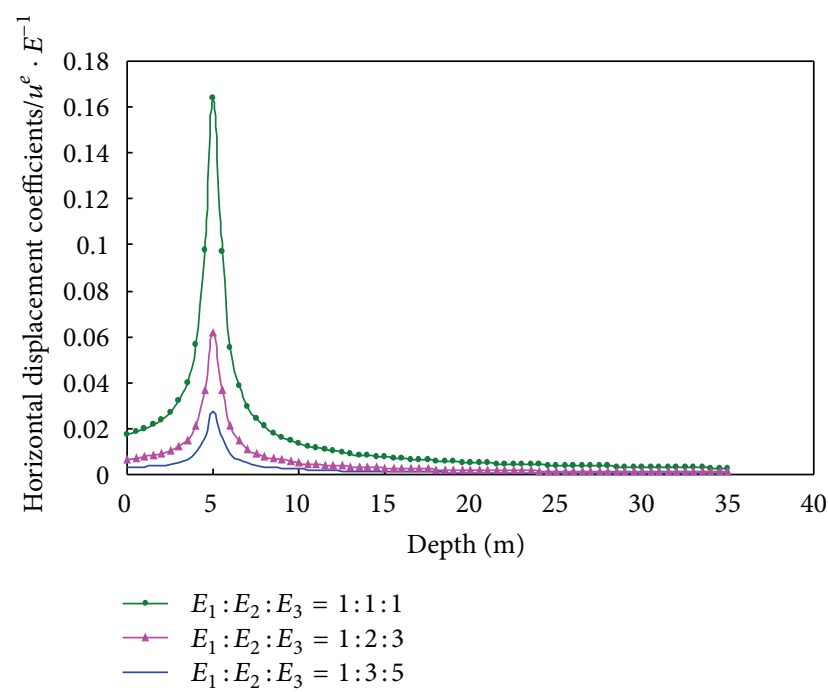

Figure 7: Comparison of the results when the subsoil modulus is larger (unit load acting at $5 \mathrm{~m}$ ).

When the three layers' soil moduli are the same, the example can be simplified as an elastic semi-infinite space problem, and a comparison can be directly made between the transfer matrix method and the Mindlin solution. As observed in Figure 5, apart from the slightly large computing results using the Mindlin Formula for the horizontal loading point, the rest points' results using the two methods are similar. Therefore, the transfer matrix method can provide solutions for elastic semi-infinite bodies by assuming that the horizontal resistance is evenly distributed around the perimeter of the pile's cross-section, which verifies the validity of this paper's method. As observed in Figure 6, the results are similar between the transfer matrix method and the finite element method. Meanwhile, the computational results of the transfer matrix method are intermediate between those of the Mindlin solution and the numerical simulation, which further proves this method's correctness.

By using the transfer matrix method, the curves of horizontal displacement coefficient are smoother than those of the Mindlin solution at the load application point and other adjacent areas, with displacement being less than the Mindlin solution. By considering the soil mass's longitudinal continuity, this paper's method manages to fully reflect the effect of the interactions between the adjacent soil layers and joint sharing of the load.

When the soil modulus is $E_{1}=E_{2}=E_{3}, E_{1}<E_{2}<E_{3}$, $E_{1}>E_{2}>E_{3}$, and $E_{2}>E_{1}, E_{2}>E_{3}$, then the corresponding horizontal displacement coefficient for unit load at a depth of $5 \mathrm{~m}$ are shown in Figures 7, 8, and 9. As observed in Figure 7, apart from the unit load's application point, the calculation of the horizontal displacement coefficient is continuous, which further proves the validity of this paper's method and corresponding program composition. Where the soil modulus is gradually increasing, following on the calculated curve's laws, the horizontal displacement coefficient will gradually decrease, and there is an obvious discrepancy in results.

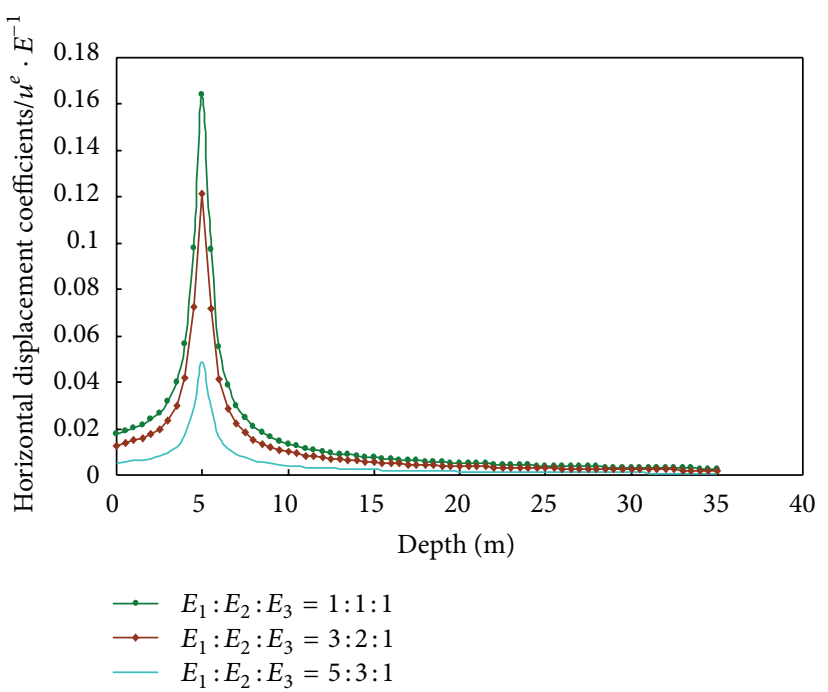

FIgURE 8: Comparison of the results when the topsoil modulus is larger (unit load acting at $5 \mathrm{~m}$ ).

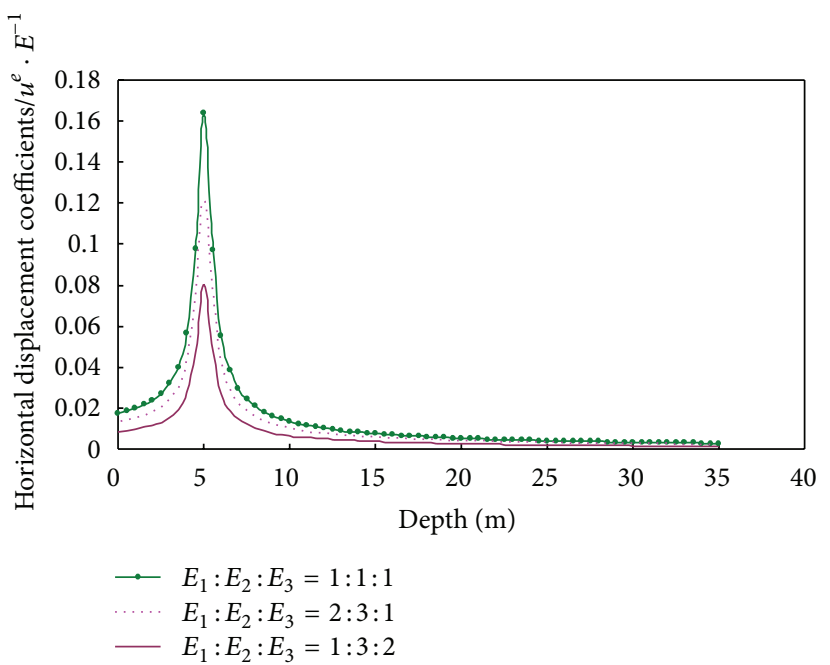

Figure 9: Comparison of the results when the interlayer soil modulus is larger (unit load acting at $5 \mathrm{~m}$ ).

According to the Mindlin formula's form, when the modulus is equal or equivalent modulus changes, the horizontal displacement coefficients for different depths will change in a fixed ratio, which cannot adequately reflect the effect brought about by the layer distribution in the layered system. As this paper's method makes clear, when the modulus is magnified, then the proportional changes to the horizontal displacement coefficient for differing depths are not the same, from the soil's surface to a depth of $5 \mathrm{~m}$, and with this change ratio in the region of $0.49 \sim 0.31$. Therefore, this paper's method is able to better reflect the effect of the layered soil's actual distribution difference.

When the soil modulus is $E_{1}=E_{2}=E_{3}, E_{1}<E_{2}<E_{3}$, $E_{1}>E_{2}>E_{3}$, and $E_{2}>E_{1}, E_{2}>E_{3}$, then the corresponding soil surface horizontal displacement coefficients for unit load acting at differing depths are shown in Figures 10, 11, and 12. 


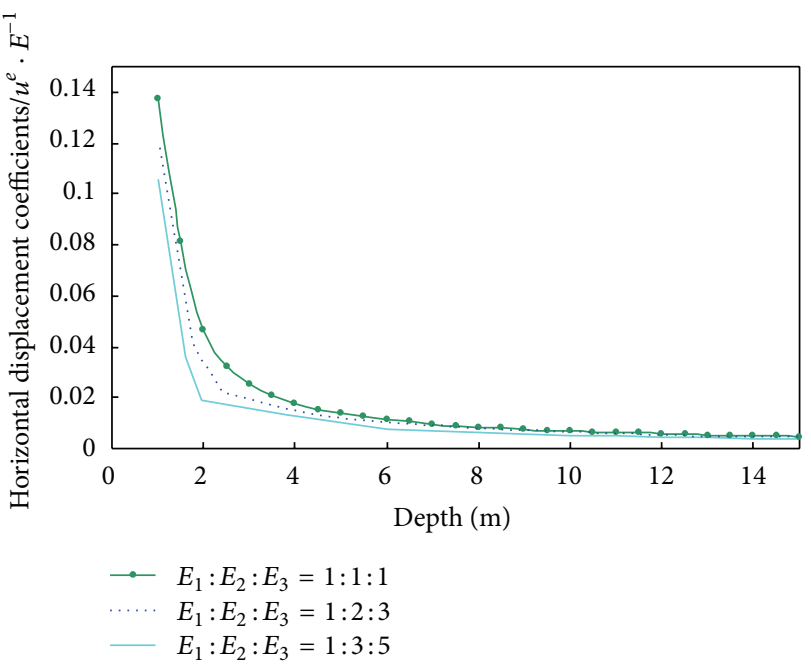

FIGURE 10: Comparison of the surface soil's results when the subsoil modulus is larger (unit load acting at different depths).

When $E_{1}=E_{2}=E_{3}$, that is, when the three layers' properties are completely equal, then the corresponding curves will be comparatively smooth. When $E_{1}>E_{2}>E_{3}$ or $E_{1}<E_{2}<E_{3}$, the change to the curve's variation trend will be relatively obvious when approaching the soil layers' interface. As the ratios of soil modulus increases the transition area of the curve increases. This change exactly illustrates that, when there are relatively large differences amongst the soil layers, then each soil layer's mechanics and load-bearing behavior will be affected by the adjacent soil layers. When $E_{1}=E_{2}=$ $E_{3}$, the result can be regarded as the calculated result of the Mindlin formula, which indicates that Mindlin formula cannot adequately reflect the effects of soil modulus variation.

\section{Conclusions}

Because the $p$ - $y$ curve method and other methods do not take into account the soil's longitudinal continuity, under relatively large horizontal loads, then amendments to the calculated horizontal displacement of soil surrounding pile need to be instituted by using the horizontal displacement coefficient matrix. Regarding the solution method for this matrix and the corrections to the soil's horizontal displacement, this paper's operations and conclusions are as follows.

(1) This paper assumed that the horizontal resistance of soil surrounding pile is evenly distributed around the pile's cross-section. According to the elastic foundation theory and layered elasticity system theory, the horizontal displacement coefficient matrix of pile foundation was calculated by the transfer matrix method. This paper's method can overcome the inconvenience and defects of applying the Mindlin formula on pile foundation. Under relatively large horizontal loads, the amended formula for horizontal displacement array $\{\mathbf{U}\}$ of soil surrounding pile, as well as horizontal displacement matrix $\left[\mathbf{u}^{e}\right]$, was calculated.

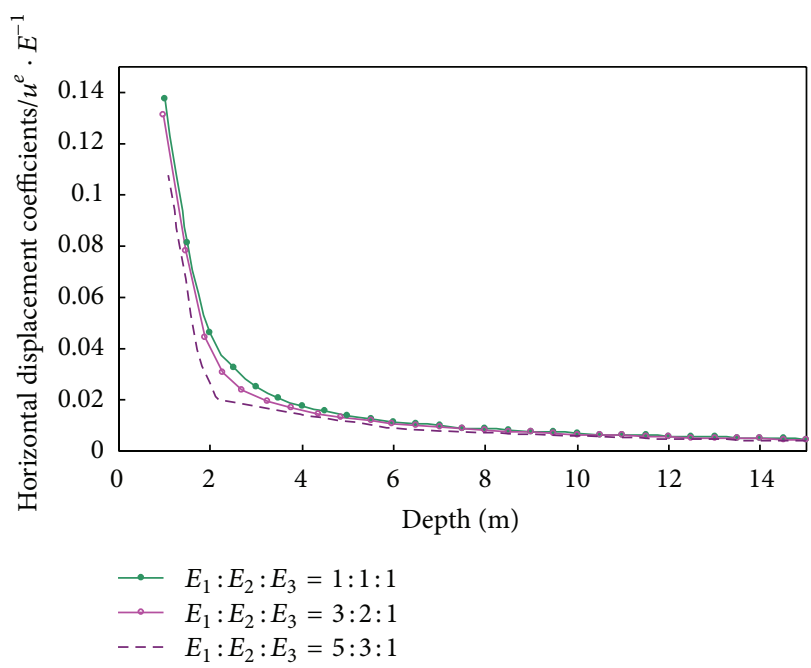

FIGURE 11: Comparison of the surface soil's results when the topsoil modulus is larger (unit load acting at different depths).

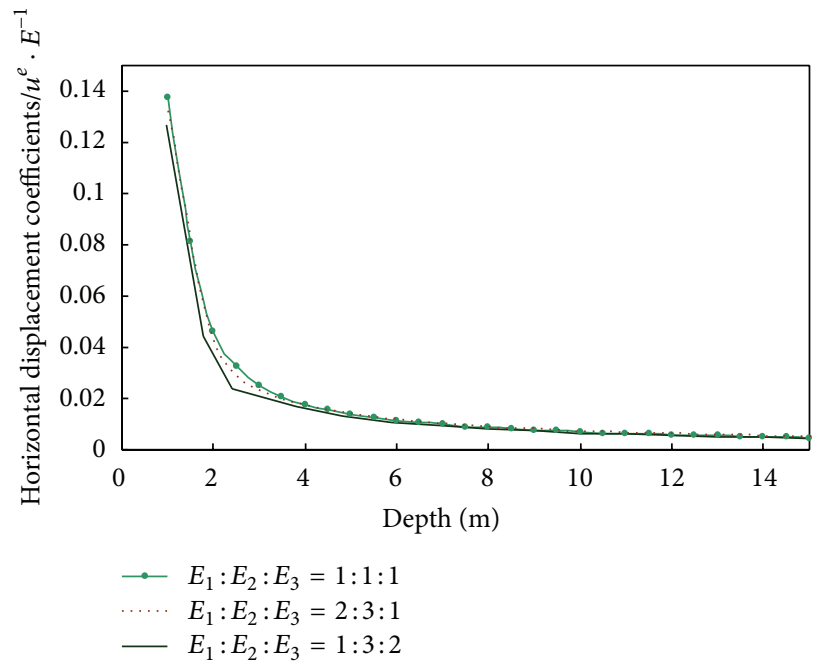

FIGURE 12: Comparison of the surface soil's results when the interlayer soil modulus is larger (unit load acting at different depths).

(2) Based on the solution model for the horizontal displacement matrix of the pile foundation, the corresponding program was worked out. When the three layers' soil moduli are the same, the layered foundations will degenerate into homogeneous foundations, and here the calculated results of this paper's model tally with those of the Mindlin formula; it is also fundamentally identical with the results of numerical simulation. These two points verify this paper's method's validity and feasibility.

(3) All the curves of horizontal displacement coefficient in this paper are smoother than the Mindlin solution's curves at the load application point and the adjacent areas, with less displacement than the Mindlin solution results.

(4) By considering the soil mass's longitudinal continuity, this paper's method is able to reflect the influence 
on the layered soils' actual distributional difference as well as the adjacent layers' interaction. It can solve the problem of the Mindlin formula being unable to adequately reflect the soil layers' distribution.

\section{References}

[1] Y. Kim and S. Jeong, "Analysis of soil resistance on laterally loaded piles based on 3D soil-pile interaction," Computers and Geotechnics, vol. 38, no. 2, pp. 248-257, 2011.

[2] G. Zheng and L. Wang, "Effect of loading level and sequence of vertical and lateral load on bearing capacity of single pile," Chinese Journal of Geotechnical Engineering, vol. 30, no. 12, pp. 1796-1804, 2008.

[3] W.-J. Yao, W.-X. Yin, J. Chen, and Y.-Z. Qiu, "Numerical simulation of a super-long pile group under both vertical and lateral loads," Advances in Structural Engineering, vol. 13, no. 6, pp. 1139-1151, 2010.

[4] W.-J. Yao, Y.-Z. Qiu, and Z.-K. Cheng, "Initial post-buckling analysis for super-long rock-socketed piles," Chinese Journal of Geotechnical Engineering, vol. 31, no. 5, pp. 738-742, 2009.

[5] W.-J. Yao, Y.-Z. Qiu, and Z.-K. Chen, "Ultimate bearing capacity analysis of a super-long rock-socketed filling pile based on catastrophe theory," Advances in Structural Engineering, vol. 13, no. 2, pp. 331-338, 2010.

[6] S. Karthigeyan, V. V. G. S. T. Ramakrishna, and K. Rajagopal, "Numerical investigation of the effect of vertical load on the lateral response of piles," Journal of Geotechnical and Geoenvironmental Engineering, vol. 133, no. 5, pp. 512-521, 2007.

[7] E. Taciroglu, C. Rha, and J. W. Wallace, "A robust macroelement model for soil-pile interaction under cyclic loads," Journal of Geotechnical and Geoenvironmental Engineering, vol. 132, no. 10, pp. 1304-1314, 2006.

[8] C. Rha and E. Taciroglu, "Coupled macroelement model of soilstructure interaction in deep foundations," Journal of Engineering Mechanics, vol. 133, no. 12, pp. 1326-1340, 2007.

[9] L.-Y. Wang, D.-X. Qian, and J.-W. Zhang, "Study on p-y curve method for computing laterally loaded piles under horizontal distribution loads," in Proceedings of the International Conference on Computer Application and System Modeling (ICCASM '10), vol. 5, pp. 366-369, October 2010.

[10] Z. K. Cheng, "Analysis of high pile structures based on p-y curve method with consideration of interaction between pile and soil," The Ocean Engineering, vol. 16, no. 2, pp. 73-82, 1998.

[11] J.-B. Su, G.-J. Shao, and N. Liu, "Nonlinear finite element analysis of piles under lateral load based on P-Y curves," Rock and Soil Mechanics, vol. 27, no. 10, pp. 1781-1785, 2006.

[12] H. G. Poulos, "Ground movements a hidden source of loading on deep foundations," Deep Foundations Institute Journal, vol. 1, no. 1, pp. 37-53, 2007.

[13] H. G. Poulos, "Behavior of laterally loaded piles: I-single piles," Journal of the Soil Mechanics and Foundations Division, vol. 97, pp. 711-731, 1971.

[14] Z. Y. Ai, Z. Q. Yue, L. G. Tham, and M. Yang, "Extended Sneddon and Muki solutions for multilayered elastic materials," International Journal of Engineering Science, vol. 40, no. 13, pp. 1453-1483, 2002.

[15] Z. Ai and M. Yang, "Extended Mindlin solution of horizontal force at a point in the interior of a layered soil," Journal of Tongji University, vol. 28, no. 3, pp. 272-276, 2000.
[16] Z. Y. Ai and J. Han, "Boundary element analysis of axially loaded piles embedded in a multi-layered soil," Computers and Geotechnics, vol. 36, no. 3, pp. 427-434, 2009.

[17] C. Ming, Studies on displacement interaction factor solution and application to composite foundation with hybrid piles [Ph.D. thesis], Shanghai Jiaotong University, 2007.

[18] F.-Q. Chen and W.-D. Huang, "A modified analysis method for passive pile based on Poulos's elastic theory," Rock and Soil Mechanics, vol. 29, no. 4, pp. 905-910, 2008.

[19] Z. Yang, W. Zheren, and G. Dazhi, “Transfer matrix method for solving non axisymmetrical problems in multilayered elastic half space," China Civil Engineering Journal, vol. 28, no. 1, pp. 66-72, 1995.

[20] L. Ga, H. Zejun, L. Weidong et al., "A precise integration approach for the dynamic-stiffness matrix of strip footings on a layered medium," Chinese Journal of Theoretical and Applied Mechanics, vol. 44, no. 3, pp. 557-567, 2012.

[21] X. Z. Lun, A Concise Course in Elasticity, Higher Education Press, 2002.

[22] G. P. Yang and Z. M. Zhang, "Research on P-Y curve of laterally bearing piles under large displacement," Port \& Waterway Engineering, vol. 342, no. 7, pp. 40-45, 2002. 


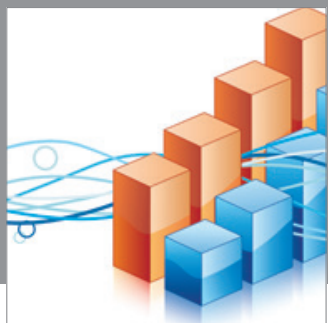

Advances in

Operations Research

mansans

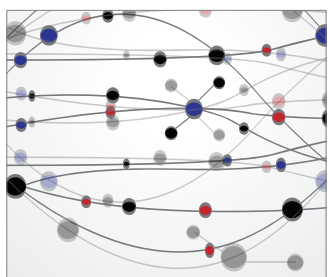

The Scientific World Journal
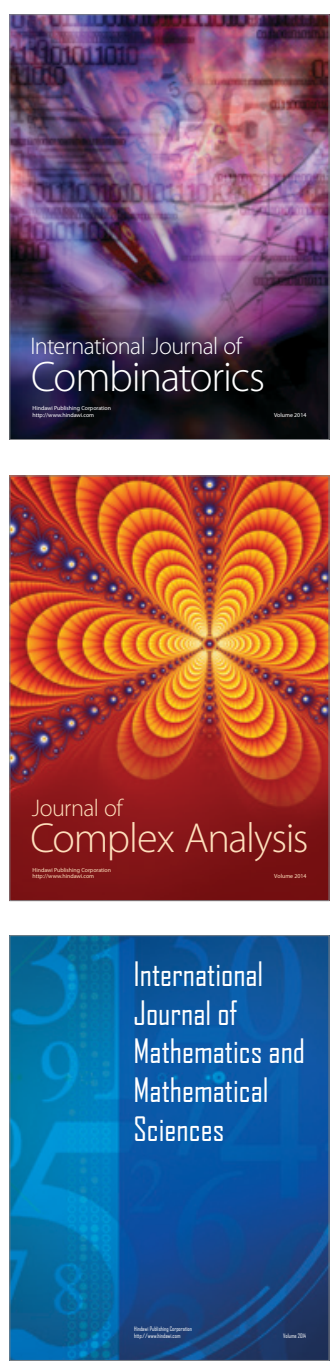
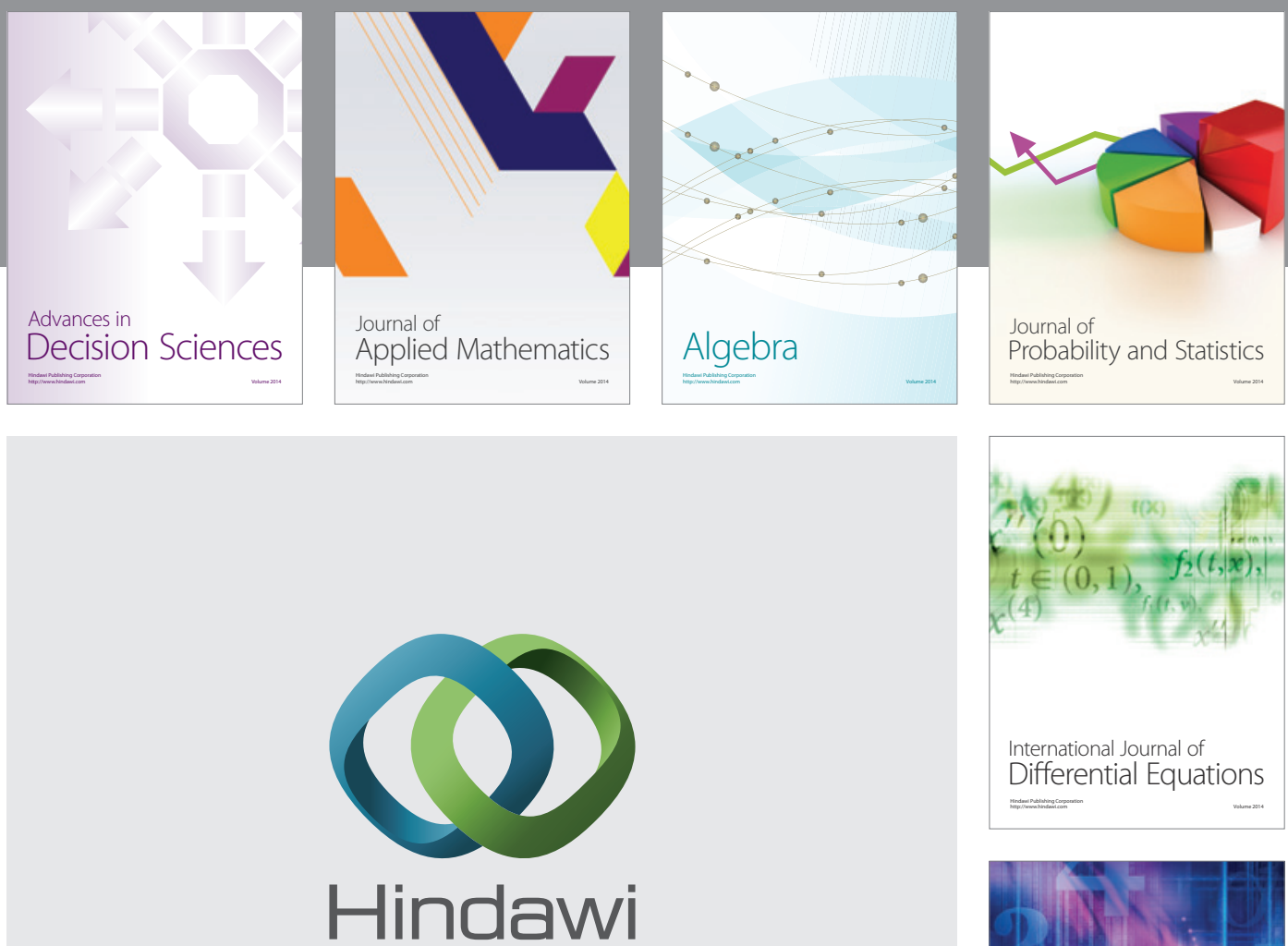

Submit your manuscripts at http://www.hindawi.com
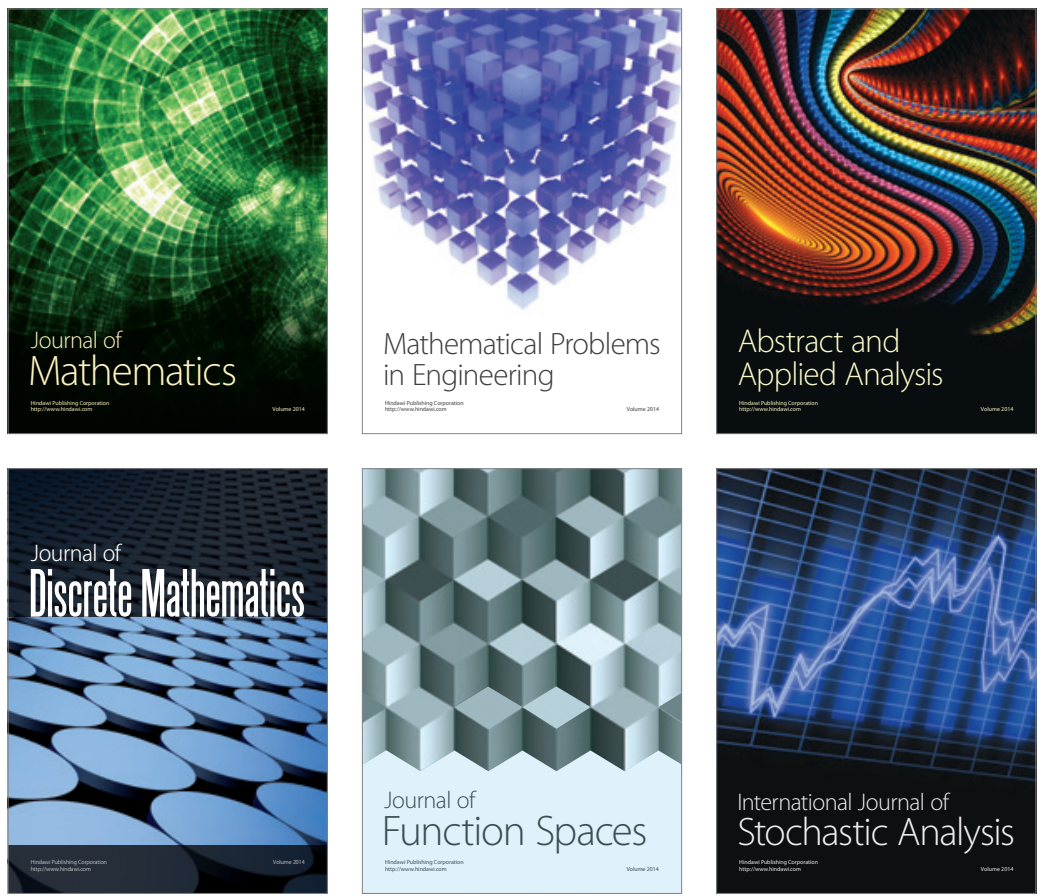

Journal of

Function Spaces

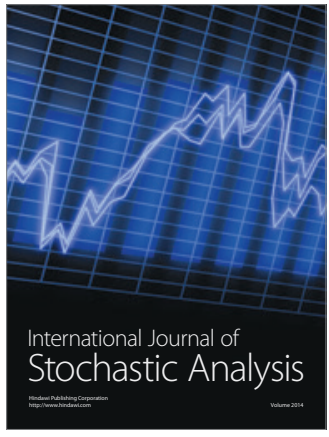

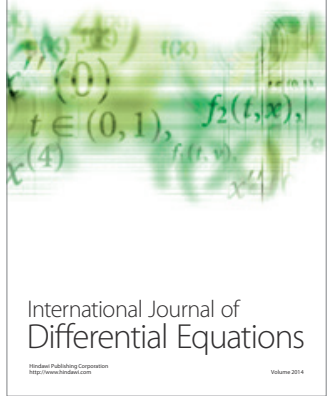
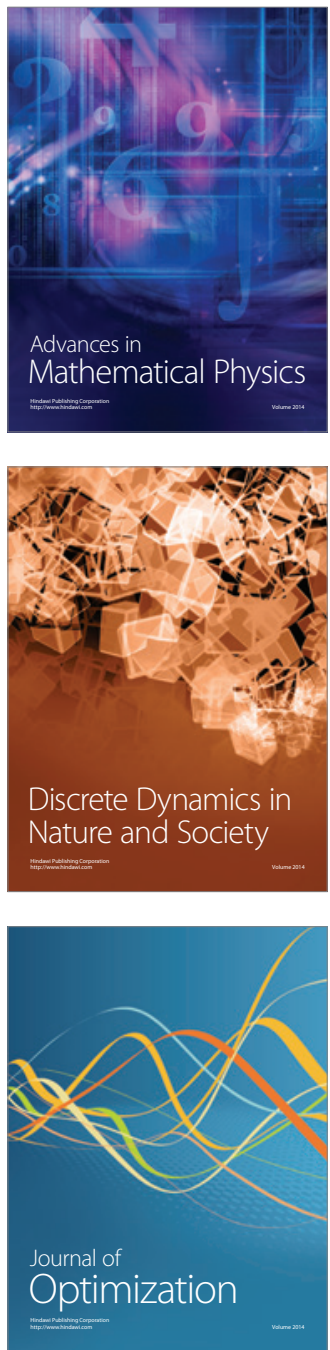